\title{
Influence des contraintes extra-linguistiques sur le discours : cas du commentaire sportif télévisé
}

\author{
Augendre, Sandra ; Mathon, Catherine; Boyé, Gilles ; Kupsc, Anna \\ CLLE-ERSS UMR 5263 \\ Augendre.Sandra@wanadoo.fr ; Catherine.Mathon@u-bordeaux3.fr
}

\section{Introduction}

L'objectif de cet article est de proposer une grille d'analyse du commentaire sportif en direct, incluant les niveaux lexical, syntaxique et prosodique. L'idée est de construire la grille d'analyse sur la base des contraintes extra-linguistiques, propres au jeu et aux tâches effectuées par les locuteurs intervenant dans le commentaire sportif en direct. Il s'agit donc, plutôt que d'établir les spécificités du discours, de relever les éléments extra-linguistiques qui vont contraindre le discours des locuteurs.

Nous utilisons comme corpus d'étude l'enregistrement du commentaire, en français, du match de rugby France-Argentine, match qui a débuté la coupe du monde de rugby 2007. L'enregistrement, d'une durée totale de 1 heure et 48 minutes, a fait l'objet d'une transcription orthographique avec le logiciel Transcriber (Barras et al., 2000). Le fichier sonore et la transcription ont été ensuite alignés avec le logiciel WinPitch Pro (Martin, 2000).

Trois locuteurs se partagent la parole durant le commentaire sportif, mais de manière inégale, et à des périodes différentes :

- Le locuteur 1 est le locuteur principal, au sens où il occupe le temps de parole le plus long. $\mathbf{3 9 \%}$ du temps d'enregistrement est constitué de tours de parole émis par le locuteur 1 . Si ce locuteur a l'expérience du commentaire sportif en direct, il n'est pas pour autant un spécialiste du rugby. Nous l'appellerons dans cet article le journaliste sportif.

- Le locuteur 2 est un spécialiste du rugby, pourtant son temps de parole est moindre que celui du journaliste sportif. Il intervient $\mathbf{2 4 \%}$ du temps d'enregistrement. Nous l'appellerons ici le spécialiste ou encore l'expert.

- Le locuteur 3 est un autre spécialiste qui se trouve sur le terrain mais dont nous n'étudierons pas les interventions, son temps de parole étant trop bref. Par ailleurs, ses interventions ponctuelles sont régulées essentiellement par le journaliste sportif.

Dans cette étude, nous montrons comment les contraintes extra-linguistiques influencent à la fois la distribution de la parole entre le journaliste sportif et l'expert mais aussi, et surtout, la nature de leurs productions.

Dans un premier temps, nous définissons le commentaire sportif, d'abord en tant que genre discursif et phonogenre, puis en tant que discours fortement contraint au niveau extra-linguistique et enfin nous présentons notre synthèse de ces différentes analyses. Nous exposons ensuite la façon dont nous envisageons une annotation de la structure et du rythme du jeu, en prenant l'exemple de l'événement touche, puis introduisons l'ensemble des contraintes extra-linguistiques que nous avons retenues comme pertinentes pour notre analyse, contraintes dont nous proposons une hiérarchisation dans le cadre de la théorie de l'optimalité. Pour finir, nous introduisons nos premiers résultats relatifs à la répartition du discours entre le journaliste et le spécialiste, en particulier au niveau du rythme, des catégories lexicales et de la syntaxe des tours de parole. 


\section{Caractérisations du commentaire sportif télévisé}

Dans le cadre de l'analyse d'un discours, quel qu'il soit, il est clair qu'il y a tout d'abord une part d'invariant, les structures syntaxiques, prosodiques ou encore lexicales étant, quoi qu'il en soit, celles disponibles dans la langue considérée. Mais tout l'intérêt de l'étude discursive, et c'est là que la notion de genre discursif prend tout son sens, réside dans les spécificités propres à un type de production, qu'il s'agisse de la fréquence d'une structure syntaxique ou prosodique, de sa fonction discursive, des choix lexicaux..., spécificités indissociables du cadre de production. C'est dans cette optique que se place notre étude du langage des médias audiovisuels, et plus particulièrement de celui du commentaire sportif télévisé, étude qui a pour but d'identifier les contraintes faisant de ce type de discours un (sous-)genre discursif à part entière.

\subsection{Le commentaire sportif en direct comme genre discursif}

Deulofeu (2000) en s'interrogeant sur la notion de genre appliquée au commentaire sportif en direct, remarque que l'on doit caractériser un genre en prenant en compte deux points de vue : un point de vue externe et un point de vue interne.

Le point de vue interne tient compte des spécificités linguistiques du genre, et dans le cadre de l'étude de Deulofeu, il s'agit de décrire les spécificités du discours au niveau syntaxique.

Le point de vue externe englobe :

- La spécificité pragmatique. Deulofeu fait remarquer que d'un point de vue pragmatique, le commentaire sportif en direct ne varie pas de manière drastique d'autres émissions médiatiques, qui impliquent l'intervention d'un animateur. Le commentateur principal peut en effet être considéré comme un animateur, qui produit une parole publique tout en essayant d'instaurer une situation de relative intimité, de « connivence» avec le public. On peut ainsi interpréter la mise en scène dialogique du commentaire comme une stratégie pour instaurer cette impression de connivence (le journaliste sportif et l'expert se présentent un peu comme des « amis » passant un bon moment devant le match) ;

- Le rôle des pratiques journalistiques. Deulofeu décrit le commentaire sportif comme «un langage technique de professionnels, où les critères stylistiques ont leur importance ».

- La spécificité cognitive. Deulofeu note que le journaliste sportif accomplit dans le commentaire des tâches cognitives multiples. Ainsi, Deulofeu distingue les tâches narrativo-descriptives des tâches explicatives. Ces dernières englobent des « commentaires, soit sur l'action qu'on vient de voir, soit sur des aspects moins immédiats du contexte (historique des équipes, de la compétition, évaluation des stratégies, etc.) ». Si les périodes explicatives ne présentent pas, selon Deulofeu, de caractéristiques très typiques, en revanche, les parties descriptives constituent une tâche « spécifique » au genre et « peu naturelle».

Sur la base de cette dernière remarque, Augendre \& Mathon (2012) reprennent cette distinction entre périodes descriptives et périodes explicatives, et tout comme Deulofeu s'intéressent particulièrement à la période descriptive en mettant en relation des éléments liés à la structure informationnelle, à l'introduction des référents dans le discours et à un découpage en macro-syntagmes. Au-delà des particularités relevées par Augendre \& Mathon (2012) sur l'introduction dans le discours des référents impliqués dans le jeu (les joueurs), il nous semble évident que les contraintes exercées sur le commentaire sportif télévisé par sa situation d'énonciation se manifestent à tous les niveaux d'analyse, aussi bien prosodique, que syntaxique ou lexical.

La distinction entre périodes descriptives et périodes explicatives au sein du commentaire sportif en direct, aussi intéressante soit-elle, se révèle parfois insuffisante pour rendre compte des données. En effet, la frontière entre les deux types de périodes n'est pas toujours clairement établie, et on remarque des 
zones de recouvrement, où les deux types sont plus ou moins représentés. Ainsi, selon la typologie de Deulofeu, le discours produit lors des ralentis appartient aux périodes explicatives. Toutefois, il nous semble que celui-ci contient une part certaine de descriptif :

regardez une fois de plus avec Skrela et là la cuiller le fameux la cuiller le petit coup du joueur en plongeant sur le talon qui déséquilibre notre buteur

La présence du déictique là, l'information fournie à la fois sur le joueur impliqué dans l'action et la dénomination de l'action sous la forme d'un nom, caractérisé par des adjectifs, des groupes nominaux apposés... Tous ces éléments relèvent selon nous autant de la description que de l'explication, ce qui remet en cause la simple dichotomie discours descriptif/discours explicatif. La différence entre le ralenti et le direct tient au type de contraintes qui leur sont associées. La description en direct, comme lors d'un ralenti offre un discours synchrone de l'action qui se déroule (qui se re-déroule) sur l'écran. En revanche, dans l'exercice du ralenti, le commentateur (qui est plus souvent l'expert que le journaliste sportif) a une connaissance déjà complète de l'action qui a été menée, de sa durée, et de ses effets sur la phase de jeu, qui s'ajoute à la lenteur du rythme propre aux ralentis. De ce fait, le commentateur a la possibilité de planifier son discours, qui, bien que synchrone, présente des caractéristiques différentes de celles du discours purement descriptif : le discours peut précéder la rediffusion de l'action, être plus développé que pour du temps réel et donc contenir des informations de type complémentaire.

Les périodes descriptives peuvent de la même manière présenter des «commentaires », des explications en marge de la description à proprement parler. Pendant une action en direct et donc au sein d'une portion de discours descriptif, certains tours de parole relèvent plus du discours complémentaire. Cependant, nous avons remarqué que ces explications sont toujours intégrées à des périodes de description d'actions de jeu relativement lentes :

\section{et ouais Mario Ledesma l'ambiance est telle dans ce stade de France il a du mal}

à comprendre les combinaisons annoncées

joue plutôt en fond de touche où Rémy Martin a essayé de batailler

Ainsi, la réalisation d'une touche, de la sortie de la balle à sa réintroduction représente un événement relativement long. La durée entre chaque phase de l'événement, et notamment entre l'installation de la ligne de joueurs pour la réintroduction de la balle et le lancer qui rendra cette réintroduction effective, permet des segments de parole plus développés et de ce fait l'intégration de périodes explicatives dans la description en direct.

En d'autres termes, l'intrusion de discours complémentaire au beau milieu d'un passage descriptif est « admis » quand l'action en cours sur le terrain est assez longue et/ou quand le déroulement et l'issue de l'action sont d'une certaine façon "prévisibles ». Entrent dans ce type d'actions les touches, dont on connaît déjà les différentes étapes (sortie du ballon, mise en place, remise en jeu) ou encore les essais, qui impliquent également certaines étapes (essai marqué, mise en place, tentative de transformation) et seulement deux issues possibles (essai transformé / essai non transformé).

Sur la base de ces observations, on ne peut que conclure que la description de la structure du commentaire sportif en direct, nécessite que soient pris en compte d'autres critères que simplement le caractère descriptif ou explicatif (commentatif) du discours, afin d'établir une typologie plus fine des différents rythmes du jeu, et des spécificités du commentaire associé. 


\subsection{Le commentaire sportif en direct comme phonogenre reconnu}

Pršir, Goldman et Auchlin (2013), dans le cadre des études en sciences du langage sur le genre, essayent d'établir une typologie de phonogenres, c'est-à-dire de genres oraux, en recoupant des situations de parole et des traits prosodiques typiques. Aucun phonogenre n'est posé a priori, mais des critères de description pragmatique des différentes situations de parole présentes dans leur corpus, permettent de premiers regroupements. Ces traits situationnels sont organisés dans quatre dimensions (les deux premières font par ailleurs écho à l'article de Deulofeu, 2000) :

- caractère médiatique

- type d'audience

- degré de préparation

- degré d'interactivité

Chaque phonogenre ainsi distingué, fait l'objet de mesures macro et micro-prosodiques, qui permettent de mettre en valeur des proximités entre les phonogenres ou au contraire des divergences, et de mesurer la distance entre eux sur la base de critères « internes » (nous reprenons la distinction ici de Deulofeu entre le point de vue externe, les traits situationnels, et le point de vue interne, les spécificités linguistiques du discours).

En ce qui concerne le phonogenre du commentaire sportif, Pršir et al. (2013) remarquent que le commentaire sportif, au sein de leur corpus composé de commentaires de trois sports différents (foot, rugby et basket), est le phonogenre le moins compact, en termes de spécificités prosodiques. Autrement dit, au sein d'un même phonogenre, on rencontre une variation prosodique importante. Cette dispersion est attribuée à « des dynamiques cinétiques différentes qui sont reflétées dans la parole».

Cette dernière remarque est basée sur une étude d'Audrit et al. (2012) qui, à l'intérieur du phonogenre du commentaire sportif, montre des différences prosodiques entre les commentaires sportifs de rugby, foot et basket, et à l'intérieur de ces commentaires entre différents types d'évènements, annotés selon la typologie suivante :

- $\quad$ context : description de faits en lien avec ce qui se passe sur le terrain.

- $\quad$ opinion : remarques faites par le commentateur qui contiennent une part de subjectivité.

- $\quad$ shot : tentative de marquer un but, qu'elle soit couronnée de succès ou non.

- possession : une équipe ou un joueur sont identifiés comme étant en possession de la balle.

- $\quad$ score : indication concernant le score.

- offence : indication concernant une infraction.

- confrontation : action offensive ou défensive d'une équipe.

- $\quad$ abort : interruption de l'action de jeu en cours.

- $\quad$ time out : rien de remarquable ne se passe.

Sur la base de cette typologie, des analyses macro-prosodiques sont menées afin d'établir des regroupements entre les différents évènements et les différents sports.

Dans la continuité d'Audrit et al. (2012), nous pensons qu'il faut distinguer différentes phases de jeu reflétées dans le discours. Pour autant, il nous semble difficile d'annoter avec le même niveau de détail ces différentes phases de jeu, sans tenir compte de la vidéo, et en les alignant avec le seul discours du commentateur. On pourrait également discuter le fait que ces annotations ne prennent pas en compte et ne reflètent pas la distribution de la parole entre les différents locuteurs qui participent au commentaire sportif. 


\subsection{Le commentaire sportif comme discours contraint}

Les quatre études que nous avons citées précédemment présentaient toutes des éléments de description majeurs du genre «commentaire sportif en direct», soit du point de vue syntaxique, soit du point de vue prosodique. Chacune de ces quatre études est partie du discours et non du jeu en lui-même, alors que chacune d'entre elles reconnaît que le jeu influence le commentaire. Deulofeu (2000) parle de critères extra-linguistiques qui doivent être pris en compte, Pršir et al. (2013) de traits situationnels qui permettent de caractériser le genre. Audrit, Pršir, Auchlin \& Goldman (2012) propose une annotation d'évènements dans le jeu et précise dans le même temps que l'annotation ne s'est faite qu'à partir du discours, indépendamment de l'image : "The tags correspond to what is said by the sports commentator, not to what occurs actually within the game $»$.

Il nous semble, quant à nous, important de considérer l'influence réelle du jeu et surtout du rythme du jeu sur le discours. Notre hypothèse est que le rythme du jeu contraint le rythme du discours, et que ces contraintes vont jouer à tous les niveaux : prosodique, syntaxique, lexical. Contrairement aux études déjà menées sur le commentaire sportif télévisé, qui admettent l'importance des paramètres extra-linguistiques sans les prendre en compte, ces contraintes sont pour nous centrales et concourent avec les contraintes linguistiques à déterminer la forme du discours.

Cette perspective permet aussi d'expliquer l'écart, souvent non négligeable, entre la durée du match et des commentaires: dans notre cas, presque un tiers du temps du match n'est pas commenté (la durée totale des tours de parole des trois commentateurs correspond à $63 \%$ du temps d'enregistrement). En effet, les locuteurs se retirent parfois afin de mettre en avant l'ambiance et les émotions du stade. Ce type de gestion de commentaires en direct est particulièrement remarquable dans les disciplines plus « esthétiques » telles que le patinage artistique ou la danse, où l'accompagnement musical joue un rôle principal dans la transmission en direct, tandis que les commentaires sont essentiellement faits pendant les ralentis pour discuter les points techniques de la présentation.

Le fait d'annoter le jeu lui-même permet d'accéder directement à la dimension synchrone des descriptions d'actions. En effet, le commentaire est contraint d'énumérer les actions qui se déroulent sur le terrain et les compléments d'informations se situent dans les interstices laissés par les arrêts de jeu et les rythmes lents.

La grille d'analyse que nous allons appliquer au commentaire sportif en direct est organisée selon trois axes :

- Les contraintes apportées par les phases de jeu. Ces contraintes sont essentiellement des contraintes rythmiques pour la forme et des contraintes informationnelles pour le fond.

- Les compétences de chacun des locuteurs. Elles ont une influence sur la structuration du discours et notamment sur la distribution de la parole. L'un des deux locuteurs, l'expert, a une compétence sur le fond: il peut donner des informations précises, mettre en perspective les stratégies de jeu. Le journaliste n'est pas un spécialiste du sport, mais il a une compétence linguistique qui lui permet de s'adapter aux contraintes imposées par le jeu.

- Une optimisation liée au genre discursif commentaire sportif. Le commentaire sportif présente une situation de «trope communicationnel» (Kerbrat-Orecchioni, 1990), c'est-à-dire que les locuteurs ont conscience de la présence silencieuse du téléspectateur, très peu mentionné dans le discours, tout en étant sa cible. Il s'agit d'un phénomène courant dans les discours médiatiques. Tout le commentaire sportif cherche à amener le téléspectateur à partager une émotion, une excitation. Cette intention de communication a une influence sur la prosodie des locuteurs, notamment du journaliste, au niveau de la F0. Sur ce point, Boulakia et al. (2009) ont montré que la volonté de transmettre de l'excitation et de maintenir l'attention du téléspectateur, amène le locuteur à utiliser une étendue de voix importante. 


\subsection{Synthèse des caractéristiques du commentaire sportif en direct}

Nous reprenons à Pršir et al. (2013) l'idée que l'on peut établir une typologie des phonogenres en fonction de critères situationnels, et parmi ceux proposés (cf. 2.2), nous retenons le caractère médiatique de la situation de parole. Le commentaire sportif télévisuel fait partie des discours médiatiques. Plutôt que d'envisager le degré de préparation du discours ou encore l'interactivité avec le public, nous avons envisagé un autre critère qui nous paraît plus saillant pour ce type de discours médiatique, le caractère « en direct », c'est-à-dire la synchronicité du discours avec le déroulement de l'événement qui fait l'objet de ce discours. De ce point de vue, le commentaire sportif peut être mis en relation avec le commentaire « en direct 》 d'autres événements médiatisés tels qu'un défilé militaire ou encore un mariage princier.

Comme Audrit et al. (2012), nous pensons que les « dynamiques cinétiques différentes » (Pršir et al., 2013) des sports ont une influence sur le discours. Par ailleurs, nous pensons également que le style du journaliste s'adapte nécessairement au sport commenté : le cent mètres, le patinage artistique et le rugby ne donnent pas lieu au même type de commentaire.

Pour prendre en compte et mesurer cette influence, nous proposons de classer les sports selon différentes catégories comme par exemple :

- Performance séparée : Les compétiteurs effectuent leur performance, seul, indépendamment des autres (exemples : patinage, bobsleigh, saut en hauteur...).

- Performance parallèle: Les compétiteurs effectuent leur performance en parallèle et simultanément aux autres (exemples : épreuve du cent mètres, aviron...).

- Combat/Match : La compétition se fait entre deux adversaires (exemples : judo et rugby). Pour les matches, le jeu est le résultat de l'interaction non seulement entre les deux équipes adverses mais également à l'intérieur de chaque équipe. C'est dans cette dernière catégorie que nous situons notre analyse.

Par ailleurs, nous pensons qu'à l'intérieur de chaque catégorie de sport, il faut prendre en compte la structure du sport en lui-même (rythme, durée, aspect esthétique...), et la décrire, pour la mettre en correspondance avec la structure du discours. Contrairement à Audrit et al. (2012), nous décidons de proposer cette description de la structure du sport, non pas en prenant pour base le discours du commentaire sportif, mais en travaillant directement à partir des images du sport. Nous considérons que chaque sport possède une structure qui lui est propre qui a un impact à la fois sur le rythme et sur le contenu du commentaire. Le type de sport pose un ensemble de contraintes sur le discours.

Un troisième ensemble de contraintes concerne les enjeux du sport, notamment le fait que l'équipe qui participe au match soit l'équipe nationale (pour les commentateurs). Rentrent également en compte dans ces contraintes l'importance de la compétition (Coupe du Monde vs compétition locale) et l'importance de la rencontre par rapport au déroulement de la compétition (quarts de finale, demi-finale, finale...). 


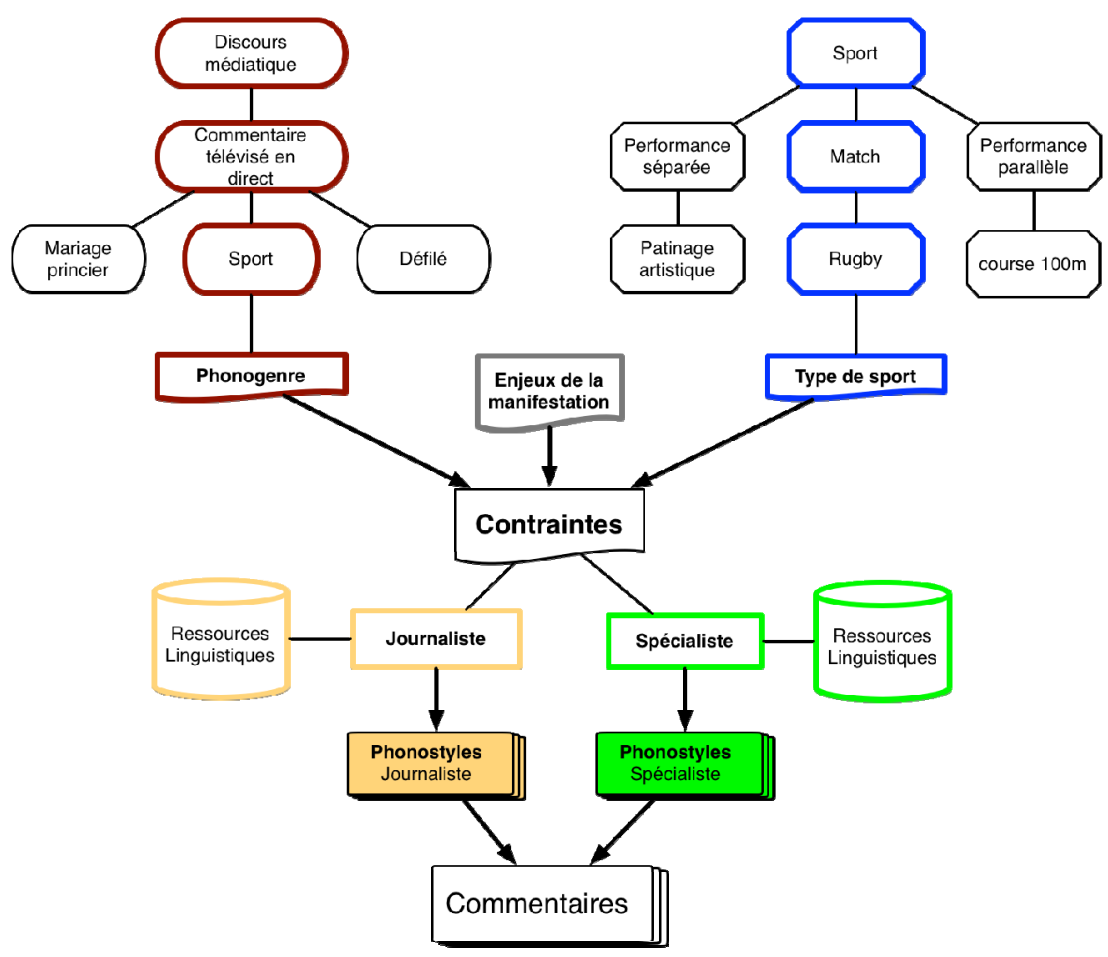

Schéma 1 : Synthèse des caractéristiques du commentaire sportif en direct

Comme le montre le schéma 1 ci-dessus, nous considérons donc que le commentaire sportif est un phonogenre, mais que le discours construit dans ce cadre répond non seulement à des contraintes liées à ce genre, mais aussi à des contraintes liées au type de sport et aux enjeux de la manifestation sportive particulière.

Comme Deulofeu (2000), nous avons noté que l'élaboration du discours était partagée par différents locuteurs (au moins deux), dont l'un est un journaliste sportif, qui intervient dans les phases de description et l'autre un spécialiste du sport, qui apporte des précisions plus techniques dans les phases complémentaires. Contrairement à Deulofeu et aux études citées plus haut, nous distinguons les productions des commentateurs, dans la mesure où elles présentent aussi des distributions structurelles et prosodiques contrastées. Nous pensons que la répartition des productions reflète des compétences linguistiques différentes. Le journaliste sportif est un locuteur spécialisé qui peut produire des énoncés très courts, efficaces pour les périodes de jeu rapides, et des modulations prosodiques très variées pour répondre aux contraintes expressives. Ceci le distingue de l'expert qui dispose de ressources linguistiques plus limitées, qui ne comprennent ni les mêmes caractéristiques expressives, ni l'aspect condensé des énoncés. Ainsi, en fonction de leurs compétences linguistiques et des contraintes (du genre, du sport et des enjeux), le journaliste et le spécialiste sont amenés à produire des énoncés caractéristiques mais différenciés sur le plan prosodique, syntaxique, et lexical. Ce sont ces différents types que nous appelons les phonostyles, constitutifs du phonogenre, commentaire sportif en direct.

\section{Identification et hiérarchisation des contraintes régissant le commentaire sportif télévisé}

Le point de départ de notre étude du commentaire sportif télévisé n'est pas linguistique mais extralinguistique. En effet, la démarche adoptée ne va pas du discours produit et de ses spécificités linguistiques vers l'identification des paramètres situationnels qui expliquent ces particularités mais au 
contraire, elle vise à partir de ces éléments extra-linguistiques à étudier la façon dont ils se manifestent linguistiquement dans le commentaire. Dans cette optique, nous avons donc commencé par identifier un ensemble de contraintes imposées par la situation d'énonciation aux locuteurs, avec l'objectif, partagé avec la théorie de l'optimalité, de hiérarchiser ces contraintes en fonction des phases de jeu et d'étudier leur influence respective sur le discours produit.

\subsection{Structure et rythme du jeu : exemple de l'évènement touche}

Nous proposons donc une analyse qui vise à rendre compte des différents rythmes des phases du jeu, qui ont une influence sur les productions des locuteurs et le choix de l'intervenant. Nous allons dans un premier temps expliciter la structure du jeu, pour ensuite voir les contraintes que cette structure impose sur le discours des locuteurs.

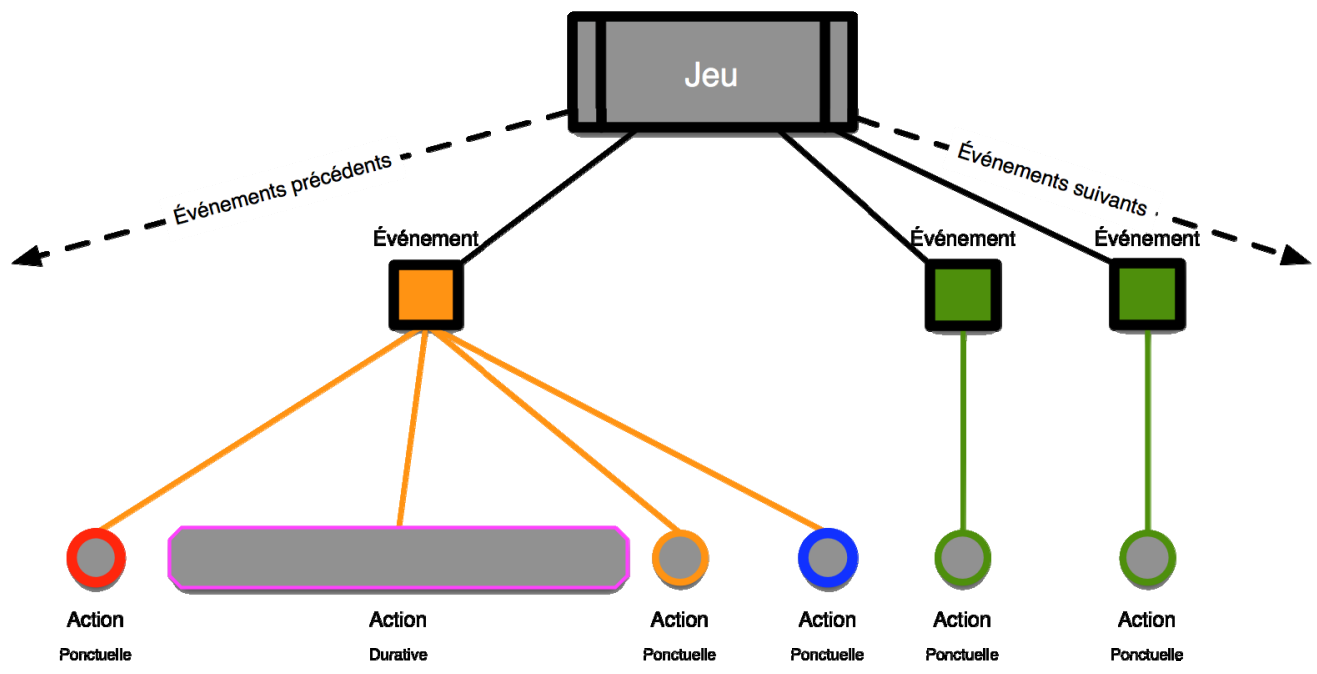

Schéma 2 : Représentation en arbre de la structure du jeu, décomposé en événements et en actions.

Le jeu est constitué d'événements, eux-mêmes décomposés en actions. Les actions peuvent être soit duratives, soit ponctuelles. Ces actions sont par ailleurs dans le jeu soit proéminentes, soit secondaires. Par proéminentes, nous entendons des actions qui vont faire systématiquement l'objet d'une mention dans le discours, car elles portent une information clef pour la compréhension du jeu. Les actions secondaires peuvent au contraire ne pas être mentionnées.

Nous allons illustrer ce premier degré de description avec un événement spécifique : la touche.

La touche par exemple est un événement qui se compose de quatre actions :

- la sortie : action ponctuelle et proéminente

- la mise en ligne : action durative et secondaire

- le lancer : action ponctuelle et secondaire

- la récupération : action ponctuelle et proéminente 


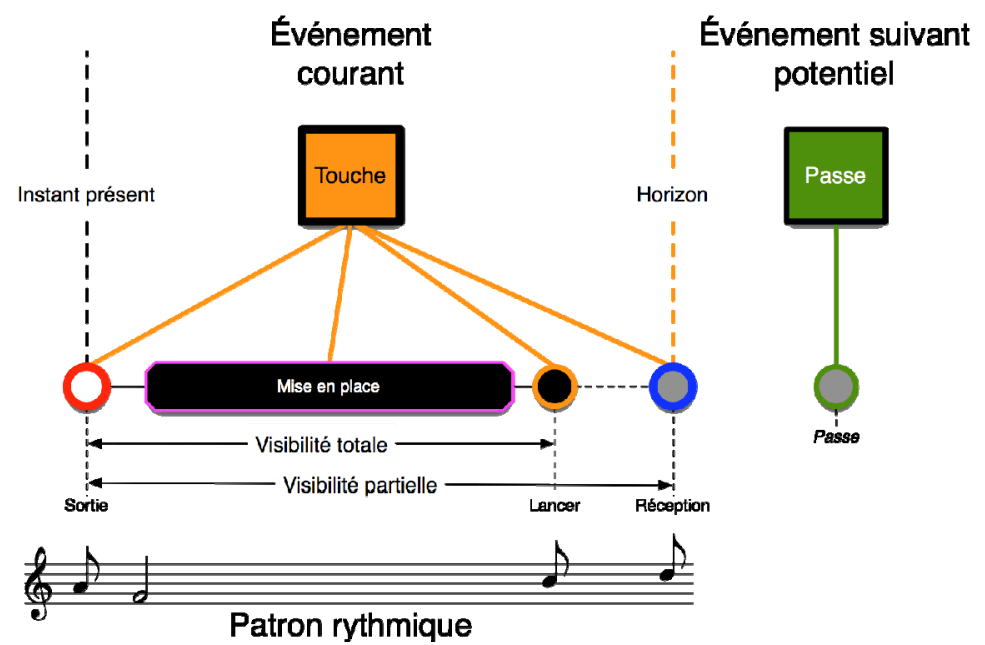

Schéma 3 : Représentation de la structure et du rythme de l'événement « touche », décomposé en actions.

Le schéma 3 représente le déroulement de l'événement touche.

Les actions ponctuelles sont représentées par des points de différentes couleurs en fonction du type de l'action, les actions duratives par des rectangles.

Les actions proéminentes sont représentées par un contour plus épais que les autres actions. Ainsi, l'action « sortie » est représentée avec un contour plus épais (en rouge) que le contour de l'action « mise en place » (en violet).

Les noyaux des cercles qui représentent les actions indiquent l'équipe en possession du ballon. Noir : Équipe A, Blanc : Équipe B, Gris : Équipe A ou Équipe B (incertitude).

La visibilité représente le temps disponible estimé avant la prochaine action (à l'intérieur de l'événement en cours). La mise en ligne (des joueurs) permet une visibilité maximale, parce que c'est une action durative avec une manifestation visuelle du progrès de l'action et de son achèvement. La visibilité partielle est due à la connaissance de la structure de l'événement et concerne une certaine incertitude par rapport au rythme exact d'enchaînement des actions.

La portée musicale permet de donner une idée du rythme associé à l'événement. La blanche représente une action durative longue, la croche représente une action ponctuelle, plus courte.

A la touche va nécessairement succéder un autre événement, noté événement suivant potentiel. La visibilité des commentateurs se limite à l'événement actuel mais ils anticipent l'événement suivant. 


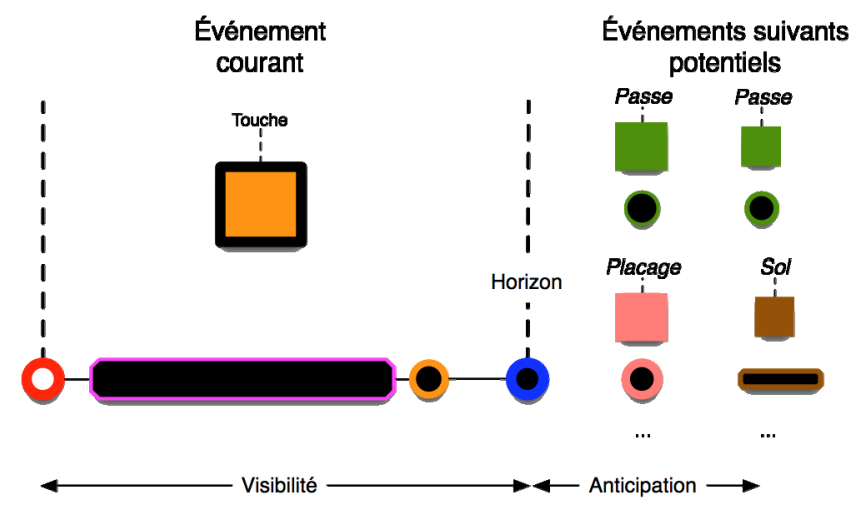

Schéma 4 : Représentation de l'enchaînement de l'événement touche avec des événements potentiels.

Au-delà de l'événement qui est en cours, le commentateur peut prévoir les événements suivants potentiels. Cette prévision est basée sur la connaissance de la structure du jeu, c'est-à-dire les enchaînements typiques d'événements d'une part, et la disposition des joueurs sur le terrain, notamment la densité de joueurs à proximité du ballon. Cette connaissance globale permet d'envisager pour l'événement suivant un certain nombre de probabilités.

La prévision de ces événements probables permet d'une part l'anticipation des patrons rythmiques à suivre, et d'autre part l'éventualité d'un événement de jeu proéminent (essai, transformation). Une telle éventualité met en jeu une des contraintes du genre commentaire sportif: la nécessité de transmettre de l'émotion. Cette nécessité contraint les patrons prosodiques utilisés par les commentateurs (rythme et mélodie).

\subsection{Hiérarchisation des contraintes dans le cadre de la théorie de l'optimalité}

L'analyse que nous présentons ici repose sur l'appareillage de la théorie de l'optimalité (Prince \& Smolensky, 1993) appliquée au domaine discursif. La parole des commentateurs est contrainte par les facteurs extra-linguistiques explicités liés au jeu et par les compétences linguistiques dont disposent les commentateurs (cf. §2.3).

Du point de vue extra-linguistique, le commentaire se doit de mentionner chaque action proéminente (ActP) et chaque événement marquant (EvM). Dans la tradition de la théorie de l'optimalité (OT), il s'agit de contraintes de fidélité entre input (jeu) et output (commentaire) que nous notons Fid(ActP) et Fid(EvM).

Pour l'influence des compétences linguistiques des commentateurs sur la prise de parole, l'analyse repose sur une comparaison entre le temps disponible avant la prochaine action proéminente (visibilité) et le temps nécessaire pour déployer les différentes structures à la disposition d'un commentateur.

D'une part, chaque intervention est limitée à la zone de visibilité et utilise une construction dont la réalisation ne dépasse pas le temps disponible *Dépas. Les interventions tendent à remplir pleinement cet espace, Max(Int). D'autre part, les interventions doivent s'appuyer sur les ressources linguistiques à la disposition de chaque commentateur. Comme nous allons l'expliciter dans la section suivante, chacun semble posséder un arsenal différent de constructions de longueurs variées, ce qui influence leurs types d'interventions possibles. Dans le cadre de l'optimalité, nous proposons d'utiliser des contraintes de 
dépendance entre les ressources linguistiques des locuteurs (input) et le discours (output). Dep(Loc) assure que les constructions utilisées par le locuteur font bien partie de son inventaire accessible.

Enfin, pour les contraintes liées au genre du commentaire sportif en direct, en plus de la contrainte * Simult qui interdit que les commentateurs parlent en même temps, nous distinguons les contraintes liées au type du contenu informationnel et celles liées à la transmission des émotions. Pour le contenu, nous avons une contrainte $\operatorname{Max}(\mathrm{Comp})$ qui garantit que le commentaire comporte le maximum d'informations complémentaires plutôt que des descriptions de ce qui est déjà visible pour les téléspectateurs. Pour les émotions, nous proposons deux contraintes :

- une qui concerne les émotions en général Max(Emo), toutes choses égales par ailleurs, le commentaire doit transmettre des émotions ;

- une autre contrainte Suspense, qui concerne les moments de tension (cf. Audrit et al. 2012), quand un événement marquant (essai, transformation critique) se situe dans la fenêtre d'anticipation, la tension monte et la prosodie change.

Nous proposons de hiérarchiser l'ensemble de ces contraintes pour régler la nature des interventions et les prises de paroles. Certaines contraintes se placent de façon évidente ${ }^{1}$ :

- $\operatorname{Dep}(\mathrm{Loc})$ domine toutes les contraintes puisqu'un locuteur n'utilise que ses propres ressources accessibles

- $\quad \operatorname{Fid}(\mathrm{EvM})$ domine toutes les autres contraintes. L'événement Essai, par exemple, a priorité sur l'intervention en cours et peut provoquer un chevauchement (Simult).

- Fid(ActP) domine Max(Comp) puisque les informations complémentaires se situent dans les temps laissés libres par les descriptions des actions proéminentes, la fidélité aux actions, en général, Fid(Act) reste inférieure aux deux autres.

- *Dépas est supérieure à Max(Int) pour que les commentaires s'adaptent au mieux au temps disponible sans le dépasser.

Le tableau ci-dessous reprend les trois situations évoquées au-dessus : un événement marquant (essai) impose une description immédiate, le jeu rapide ne laisse pas la place au discours complémentaire, le ralenti au contraire demande des interventions étoffées.

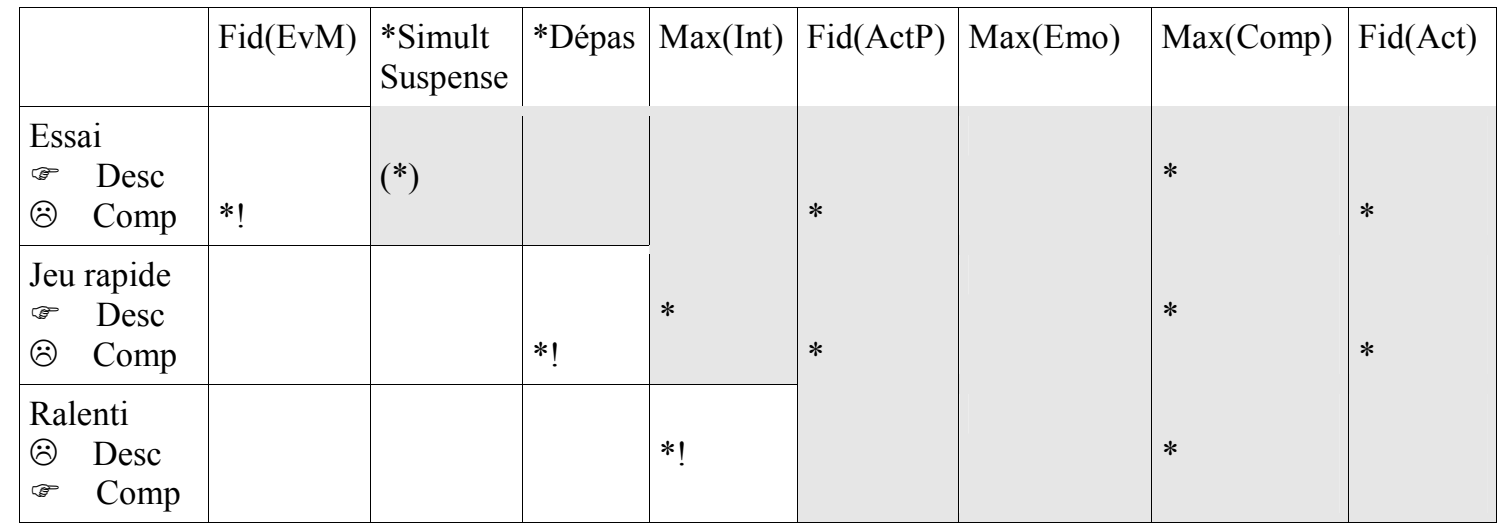

Tableau 1 : Exemples d'interactions entre contraintes : essai, jeu rapide, ralenti.

Pour la première ligne, par exemple, on interprète le tableau comme suit. Placer du discours complémentaire (Comp) au moment d'un essai (événement marquant) contrevient à la contrainte de fidélité la plus importante Fid(EvM), c'est cette infraction qui est décisive (*!) dans la sélection ( ) d'une séquence descriptive (Desc) et le rejet ( ) de la complémentaire. Dans cette situation, il est même possible d'interrompre le discours de l'autre commentateur pour décrire l'essai puisque la contrainte de non-simultanéité, *Simult, est inférieure à celle qui demande qu'on décrive les évènements marquants 
Fid(EvM). Ne pas décrire l'essai contreviendrait également aux contraintes de fidélité aux actions proéminentes, Fid(ActP) et à la contrainte de fidélité aux actions en général, Fid(Act). De son côté, la description de l'essai viole la contrainte de maximisation du discours complémentaire, Max(Comp) mais elle est nettement inférieure à la contrainte Fid(EvM) et sa violation ne joue pas de rôle dans la décision (zone grisée).

\section{Différenciation entre commentateurs : journaliste vs spécialiste}

Sur la base de la description de la structure et du rythme du jeu d'une part, et des contraintes énoncées en 3.2., nous avons procédé à quelques analyses préliminaires permettant de mettre en perspective l'influence du rythme de jeu et des ressources linguistiques sur la structuration du discours. En l'absence d'une annotation complète du corpus (vidéo et discours) prenant compte de la structure du jeu, nous avons tenu compte dans nos analyses d'une annotation antérieure en périodes descriptives et périodes complémentaires, conscients toutefois que cette annotation présentait les défauts énoncés plus haut (cf. 2.1.).

Il nous a semblé plus important de nous concentrer sur la distinction entre le journaliste et l'expert. En effet, si l'annotation en périodes descriptives et complémentaires est discutable, l'annotation des locuteurs en fonction des tours de parole est stable, et ne changera pas, quelle que soit la perspective adoptée pour l'analyse des données.

\subsection{Différenciation en terme de rythme}

Nous avons dans un premier temps examiné la distribution des interventions du journaliste et du spécialiste par tours de parole, en termes de nombre, de durée, de syllabes et de mots. La figure 1 présente le nombre de tours de parole par rapport au découpage en périodes descriptives et périodes commentatives (explicatives) sur le total des tours de parole, et par locuteur.

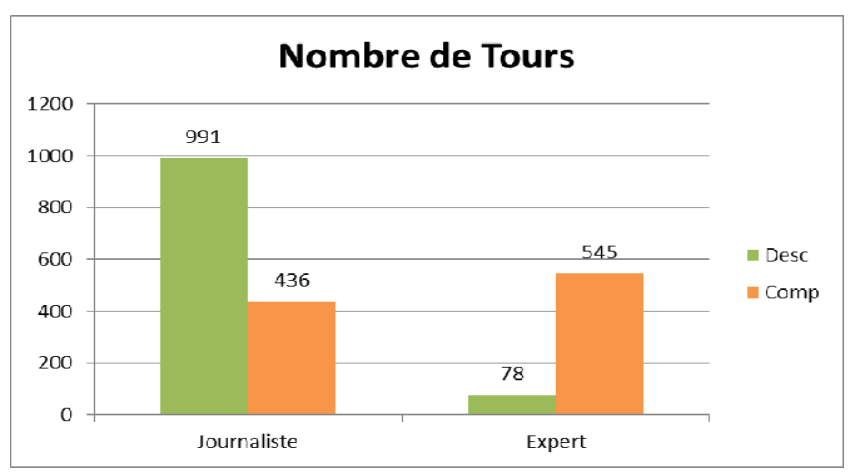

Fig. 1 - Nombre de tours par type de discours par locuteur

Chaque locuteur semble associé à un type de discours et donc à un rôle au sein du commentaire : le discours descriptif est constitué à plus de $90 \%$ d'interventions du journaliste, alors que le discours complémentaire, bien que mieux distribué, relève plutôt du spécialiste, qui y produit plus de $55 \%$ des interventions. Le lien existant entre le type de discours et chaque locuteur est nettement observable si l'on considère la répartition des prises de parole de chacun des locuteurs : si $70 \%$ des interventions du journaliste sportif appartiennent au discours descriptif, 87,5\% des interventions du spécialiste relèvent du discours complémentaire. 


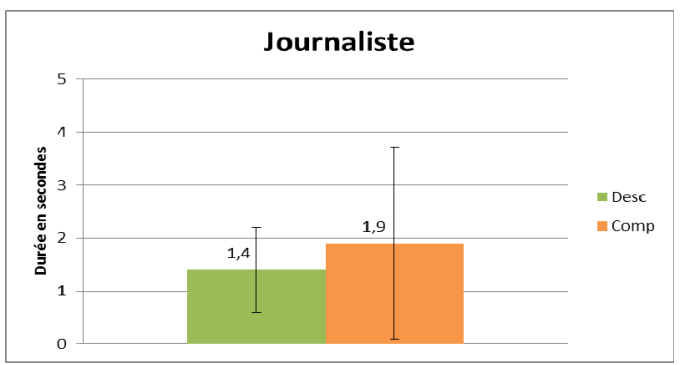

Fig. 2 - Durée moyenne des tours de paroles du journaliste en secondes

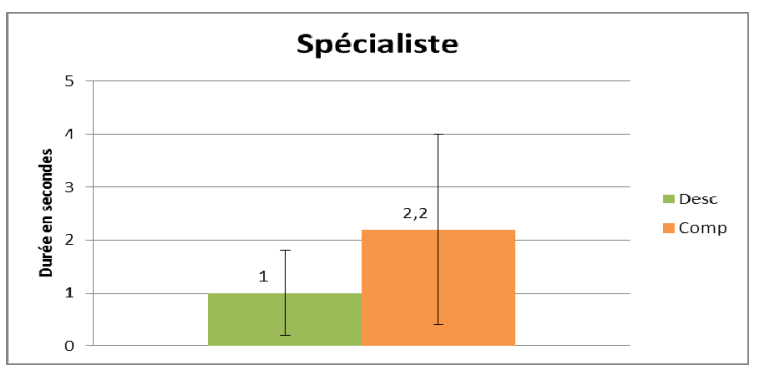

Fig. 3 - Durée moyenne des tours de paroles du spécialiste en secondes

Les figures 2 \& 3 présentent la durée moyenne (en secondes) des tours de parole du journaliste et du spécialiste en fonction des périodes, descriptive (en vert) ou complémentaire (en orange). On observe pour le journaliste sportif (cf. Fig.2), que la différence de durée entre les tours descriptifs et les tours complémentaires n'est pas très importante, tandis que pour le spécialiste, la durée des tours de parole complémentaires est deux fois plus longue que celle des tours descriptifs.

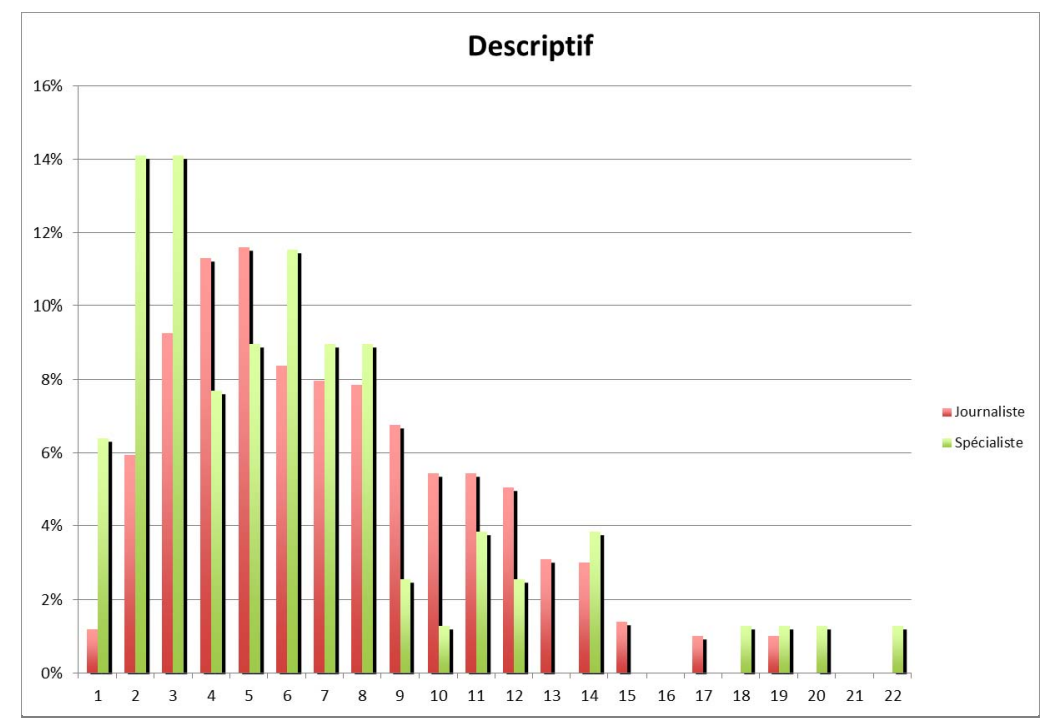

Fig. 4 - Distribution du nombre de syllabes par tour dans les descriptions 


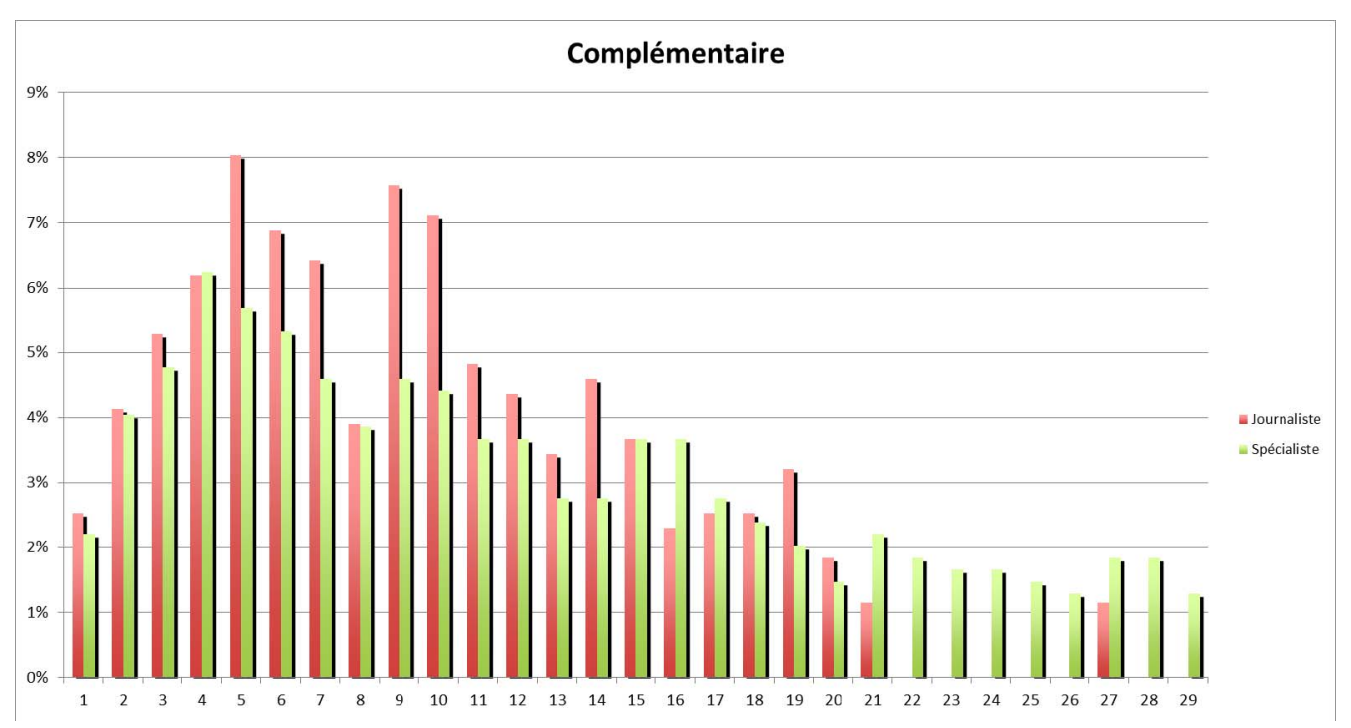

Fig. 5 - Distribution du nombre de syllabes par tour dans les compléments

Les figures $4 \& 5$ présentent la distribution du nombre de syllabes par tour dans les périodes descriptives (Fig. 4) et les périodes complémentaires (Fig. 5). On remarque que dans les périodes descriptives, les tours courts, de 2 à 9 syllabes par tour, sont prépondérants. Par ailleurs, on note que le journaliste (en rose) a une palette plus étendue, là où la distribution des longueurs des tours de parole de l'expert est plutôt chaotique.

Dans les périodes complémentaires au contraire, les tours longs, de 2 à 15 syllabes par tour, sont plus fréquents. Les tours de parole du journaliste sont à peu près de même étendue que dans les périodes descriptives, tandis que l'expert produit des tours beaucoup plus longs.

Nous avons observé également la longueur en mots des tours de parole en fonction des locuteurs et du découpage discours descriptif / discours complémentaire.

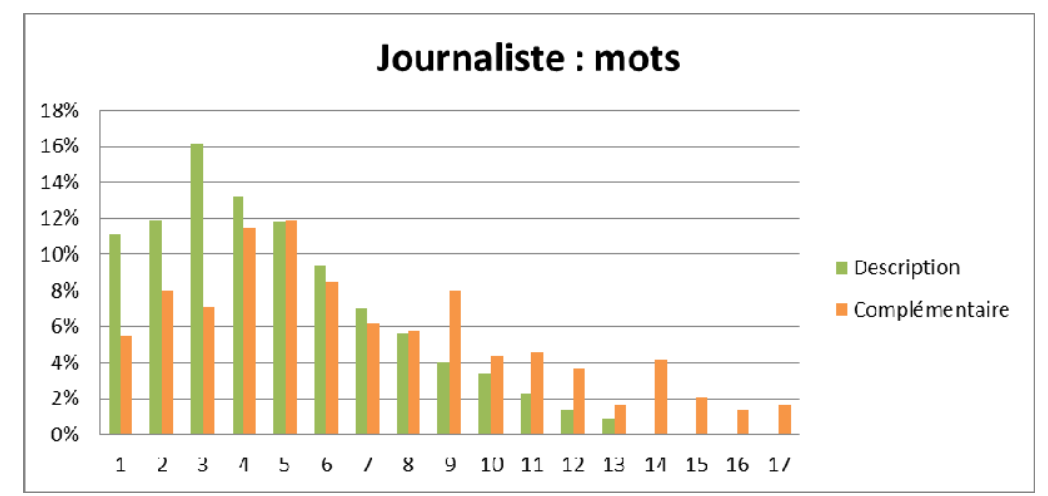

Fig. 6 - Distribution du nombre de mots par tour et par période dans le discours du journaliste 


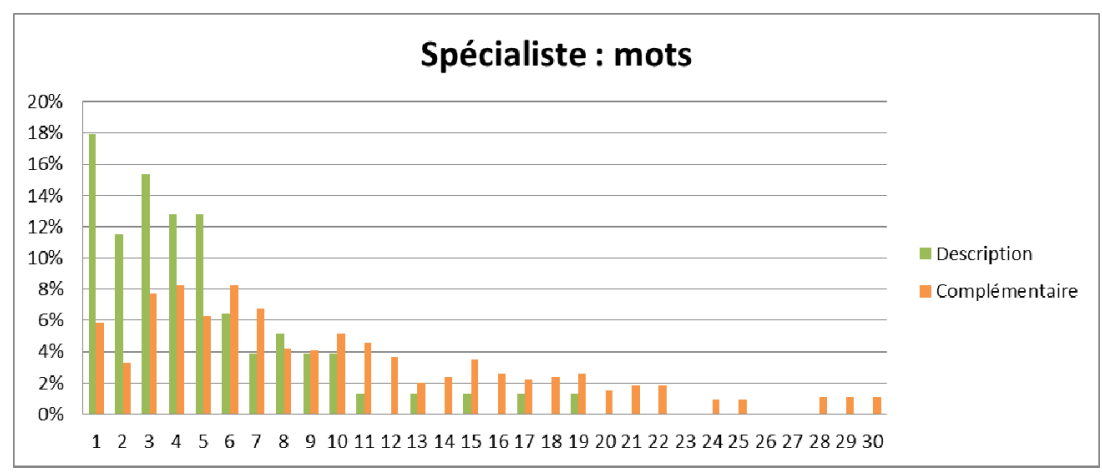

Fig. 7 - Distribution du nombre de mots par tour et par période dans le discours du spécialiste

Indépendamment du locuteur, la période descriptive présente des tours de parole essentiellement courts. Le journaliste utilise une palette plus large et a une distribution plus régulière dans cette zone que le spécialiste, qui utilise essentiellement des tours très courts de façon erratique. A l'inverse, pour la partie complémentaire, les tours de parole sont plus longs, quel que soit le locuteur. Cette fois-ci, le spécialiste a la plus grande variété de nombre de mots.

Au niveau du rythme, on a observé une différenciation importante, tant au niveau de la durée des tours que du nombre de syllabes et de mots, entre spécialiste et journaliste et aussi pour un même locuteur entre discours descriptif et discours complémentaire.

\subsection{Différenciation en termes de ressources lexicales}

Nous nous sommes d'abord intéressés à l'usage des noms et verbes par les deux locuteurs. Les tableaux cidessous présentent la distribution des noms et des verbes par locuteur (journaliste et spécialiste), et par type de période.

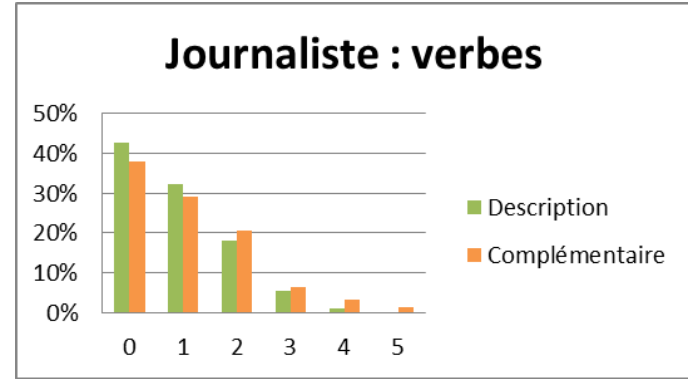

Fig. 8 - Distribution du nombre de verbes par tour et par période dans le discours du journaliste

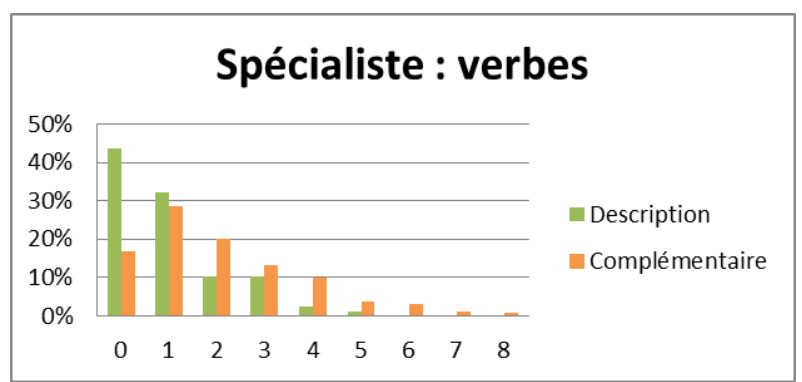

Fig. 9 - Distribution du nombre de verbes par tour et par période dans le discours du spécialiste

On voit que le journaliste n'utilise pas de verbe, indépendamment du type du discours, pour environ 40\% de ses interventions, tandis que le spécialiste utilise deux fois plus fréquemment les constructions sans verbe dans la partie descriptive (plus de 40\%) que dans le discours complémentaire (moins de 20\% de tours). Par ailleurs, la distribution du nombre de verbes par tour pour le journaliste est linéairement décroissante de 0 à 5, dans les deux types de discours. En revanche, dans le discours complémentaire, le spécialiste utilise plus de verbes (jusqu'à 8) et plus souvent. La distribution des verbes dans la description est semblable pour les deux locuteurs. 


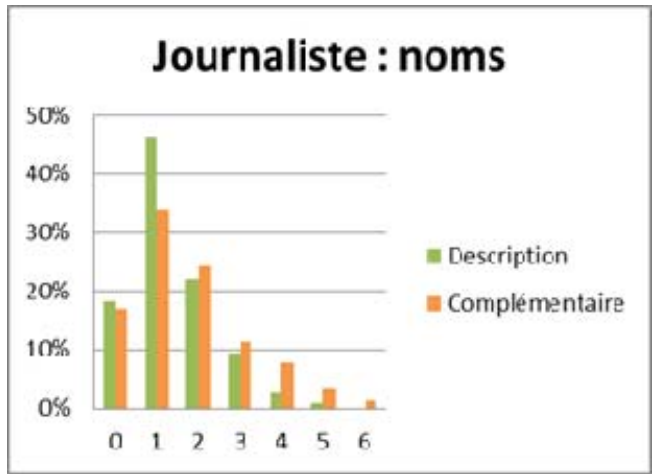

Fig. 10 - Distribution du nombre de noms par tour et par période dans le discours du journaliste

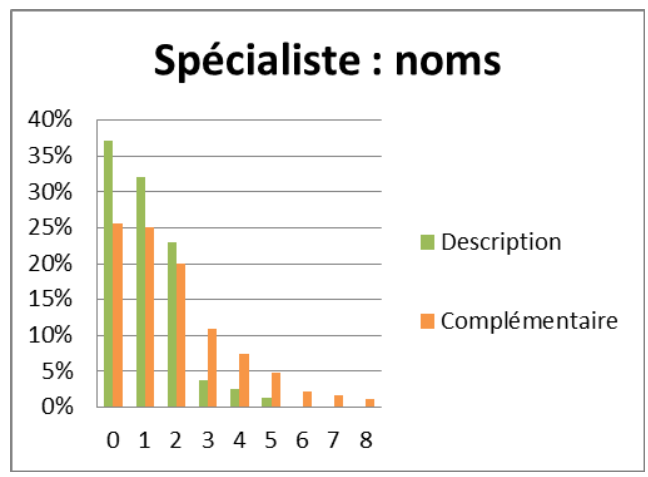

Fig. 11 - Distribution du nombre de noms par tour et par période dans le discours du spécialiste

Les interventions anominales sont beaucoup plus présentes chez le spécialiste : dans le discours descriptif il en utilise presque 2 fois plus (35\% vs. 18\%) tandis que dans le discours complémentaire la proportion est de 25\% contre 18\% pour le journaliste. Dans l'ensemble, la distribution des noms est plutôt caractéristique du locuteur que du type de discours.

Ces observations confirment que les deux locuteurs n'emploient pas les même stratégies linguistiques, les constructions poly-verbales sont plus caractéristiques du spécialiste, et que le discours descriptif favorise les interventions courtes, donc averbales. De fait, les interventions du journaliste contiennent globalement plus de noms et celles du spécialiste plus de verbes.

Ces résultats, reliés à la répartition des interventions des deux locuteurs, permettent de mettre en relation les locuteurs (journaliste vs. spécialiste), leur savoir-faire respectif (aller à l'essentiel vs. développer des idées), le type de discours produit (décrire vs. informer) et la forme qu'il prend (énoncés courts à tendance nominale vs. énoncés (plus) longs à tendance multi-verbale).

\subsection{Différenciation en termes de ressources syntaxiques}

Après ces considérations lexicales, nous allons observer plus en détail la distribution de quelques structures syntaxiques entre le journaliste et l'expert dans notre corpus, à partir de la liste proposée par Deulofeu (2000).

Sans tenir compte du cadre macro-syntaxique de l'étude de Deulofeu, nous avons choisi de lui reprendre quatre patrons structurels, afin d'en analyser la fréquence et la distribution entre les locuteurs au sein de notre corpus.

\section{Noms propres isolés}

Dans notre corpus, la centaine de tours de ce type appartient pour la quasi-totalité au discours descriptif du journaliste avec seulement 4 interventions du spécialiste, qui se résument à un nom propre ou à une séquence prénom + nom.

Un énoncé limité à un nom propre est donc un premier patron syntaxique typique du journaliste qu'il utilise quand il dispose d'un temps de parole extrêmement limité et qu'il doit réduire son discours à la description des actions proéminentes qui se succèdent rapidement (*Depas et Fid(ActP)). 


\section{Syntagmes prépositionnels sans élément recteur}

Ce deuxième type de structure est introduit comme unité autonome sans élément recteur, comme par exemple dans l'enchaînement de tours suivant :

C'est une bonne séquence

Avec Skrela

Avec Rougerie

Comme les tours correspondant au nom propre isolé, le syntagme prépositionnel sans élément recteur ressort comme étant un des patrons syntaxiques caractéristiques du journaliste, qui l'utilise 7 fois plus souvent que l'expert (111 occurrences pour le journaliste contre seulement 15 pour l'expert). Ce type de tour, indiscutablement lié au format court, lui permet de se passer d'un groupe verbal. La préposition fournit des informations sur le déplacement du ballon ( «avec» par exemple, indique le (nouveau) possesseur du ballon et « pour » le destinataire).

\section{Structures Nom + «qui » + verbe}

La structure peut paraitre complexe car elle contient une subordonnée, mais elle est essentiellement nominale et syntaxiquement autonome.

Le résultat des analyses effectuées sur la séquence Nom + «qui » + verbe montre que son statut nominal, son caractère synchrone à l'action et le fait qu'elle permette d'introduire deux informations nouvelles, en font un patron syntaxique efficace dans les formats courts. Ainsi, le journaliste utilise 97 fois ce type de structure alors que le spécialiste ne l'utilise que 36 fois, c'est-à-dire 3 fois moins souvent.

Au-delà de sa complexité, la structure présente un ensemble de propriétés qui en font une bonne candidate pour les descriptions rapides. Prenons un exemple afin d'illustrer nos propos :

Harinordoquy qui conserve le ballon dans ses pieds

Dans ce tour, produit en tant qu'ensemble syntaxiquement et informationnellement autonome, le point de départ est un nom propre fournissant la seule information nécessaire à cet instant du match : l'identité du possesseur du ballon. Dans la relative qui en dépend aux niveaux syntaxique et référentiel, le journaliste reprend cette information mais en tant que déjà partagée (avec le relatif), ce qui lui permet d'introduire un deuxième bloc d'informations nécessaires (le ballon est conservé dans les pieds).

\section{Structures « avec X qui »}

Cette structure fusionne le patron Syntagme prépositionnel sans élément recteur avec la structure Nom + qui + verbe. Dans notre corpus, cette construction n'est pas fréquente ( 4 occurrences pour le journaliste et 2 occurrences pour l'expert). Voici un exemple :

avec Scelzo qui a laissé pour euh Pichot

Les structures «avec X qui » reprennent les propriétés des deux patrons fusionnés. Elles forment à la fois des syntagmes autonomes et informationnellement suffisants, appropriés pour les formats courts. Ces structures permettent d'introduire deux blocs d'informations nouvelles, dans le syntagme prépositionnel et dans la relative. Dans l'exemple ci-dessus, le commentateur indique d'abord un changement de possesseur (en utilisant «avec») et fournit une première information essentielle : l'identité du nouveau possesseur («Scelzo »). La reprise pronominale (« qui »), qui relègue immédiatement « Scelzo » au rang d'information d'arrière-plan, montre que le ballon est déjà ailleurs et que le discours doit se recentrer («pour ») sur le nouveau possesseur («Pichot»), focus informationnel de la deuxième partie du tour de parole. 


\section{Une structure émergente : la dislocation à droite}

Au-delà des patrons syntaxiques relevés par Deulofeu, nous avons remarqué la présence massive de dislocations à droite de noms propres, du type :

\section{il est pris Rémy Martin}

La dislocation à droite est une structure syntaxique dans laquelle un référent de discours est introduit deux fois au sein d'un même énoncé, la première fois sous la forme d'un clitique («il » dans l'exemple) et la deuxième fois sous la forme d'un syntagme plein («Rémy Martin» dans l'exemple). Cette construction est généralement considérée comme typique de l'oral et beaucoup moins fréquente que la dislocation à gauche, où un syntagme plein est suivi d'une reprise clitique : selon l'étude sur un corpus d'entretiens d'Ashby (1988: 206-207), la dislocation à gauche est environ dix fois plus fréquente que la dislocation à droite. Par ailleurs, au niveau informationnel, la fonction la plus souvent attribuée au syntagme isolé syntaxiquement (et souvent prosodiquement) en fin d'énoncé est celle de rappel (du thème) permettant de clarifier ou désambiguïser le propos (cf. Apothéloz, 1997).

Un comptage rapide des dislocations impliquant les noms propres de notre corpus, révèle une tendance inverse à celle constatée dans les travaux cités ci-dessus, avec presque deux fois plus de dislocations à droite que de dislocations à gauche (31 contre 17). Par ailleurs, nous avons noté que si la dislocation à gauche est un patron syntaxique, typique de l'oral, et qui se trouve donc à disposition à la fois du journaliste (9/17) et du spécialiste (8/17), la dislocation à droite relève principalement du discours descriptif du journaliste (24/31). Enfin, au niveau informationnel, les noms propres disloqués en position postverbale n'assument pas une simple fonction de rappel mais sont majoritairement des référents nouveaux présents dans le contexte situationnel immédiat, celui du match.

Indépendamment du statut de l'élément disloqué, la dislocation à droite permet d'introduire d'abord les données relatives à l'action puis celles relatives au(x) participant(s), organisation de l'information largement sollicitée par les intervenants, que l'on retrouve par ailleurs dans les énoncés nominaux du type «nom (action) de nom propre» (par exemple : «récupération de Pichot»). La présence massive de dislocations à droite est en grande partie imputable à une contrainte extralinguistique : l'action est directement visible pour le téléspectateur, alors que l'identification du joueur n'est pas toujours immédiate. Par ailleurs, la dislocation à droite permet certaines libertés : l'élément disloqué étant déjà représenté dans l'énoncé par un clitique, le locuteur a la possibilité de l'insérer après le verbe, s'il en a le temps, mais n'y est pas forcé (l'énoncé reste complet).

Globalement, les analyses que nous avons réalisées sur la syntaxe des tours de parole de notre corpus ont mis à jour un ensemble de patrons très fréquents dans le discours descriptif, mais qui sont aussi et surtout utilisés par le journaliste, quel que soit le discours qu'il produit. Les principales structures syntaxiques typiques de son discours sont donc les suivantes: des énoncés courts à tendance averbale et des syntagmes nominaux et prépositionnels autonomes qui introduisent un référent et qui sont éventuellement complétés par des subordonnées relatives en «qui », qui permettent d'introduire les actions associées à ces référents : une palette qui permet d'adapter le discours descriptif à différentes situations de visibilité.

\section{Conclusion}

Notre article propose une analyse du commentaire sportif en direct qui part d'une analyse du jeu, et non simplement du commentaire. Il nous semble en effet que pour montrer l'importance des contraintes que l'organisation et le rythme du jeu exercent sur le discours des commentateurs, il faut avant toute chose, décrire ces paramètres.

Nos premières analyses ont montré une réelle différence entre le journaliste et l'expert quant à :

- La distribution de leurs interventions dans les périodes descriptives et complémentaires. L'essentiel des tours de parole du journaliste est concentré dans les périodes descriptives. Plus 
intéressant encore, le journaliste n'est pas exclusivement cantonné aux périodes descriptives. Les deux commentateurs interviennent de manière relativement équilibrée dans le discours complémentaire.

- La longueur des structures qu'ils sont amenés à produire, qu'elle soit mesurée en secondes, en nombre de syllabes par tour ou encore en nombre de mots par tour. On note que le journaliste dispose d'une grande variété de constructions permettant de s'adapter à toutes sortes de tours courts, tout en étant capable de former, quand les contraintes le lui permettent, des structures plus longues. Réciproquement, le spécialiste dispose d'une palette large pour intervenir dans des tours longs, mais peut produire accessoirement quelques descriptions courtes.

Le fait de partir d'une analyse du jeu, et non directement d'une analyse du discours, nous a permis d'envisager le commentaire comme un discours sous contraintes :

- Contraintes extra-linguistiques apportées par le jeu (Fid(EvM), Fid(ActP)) ;

- Contraintes liées aux compétences linguistiques à disposition des locuteurs (Dep(Loc), *Dépas) ;

- Contraintes liées au genre discursif et au phonogenre (Max(Emo) et Suspense).

L'élaboration d'une structuration détaillée du jeu a pour but de mieux rendre compte d'une part des contraintes rythmiques et informationnelles apportées par les phases de jeu, Fid(ActP), Fid(EvM), et d'autre part des compétences de chacun des deux locuteurs, Dep(Loc). La prise en compte de ces contraintes englobe également la distinction entre caractère synchrone ou asynchrone du discours par rapport à l'image, ainsi qu'une meilleure caractérisation des périodes mixtes comme les ralentis.

La suite de notre travail portera sur l'analyse des ressources de chacun des locuteurs aux niveaux prosodique, syntaxique et lexical. L'intégration des contraintes permet de prédire dans une certaine mesure la distribution des interventions du journaliste et du spécialiste. L'objectif de nos analyses futures sur les trois plans, prosodique syntaxique et lexical est d'être en mesure de prédire à termes non seulement la distribution de la parole mais aussi la distribution de la structure des contenus : mobilisation des ressources en fonction des phases de jeu (constructions syntaxiques, patrons mélodiques et vocabulaire).

Au niveau syntaxique, nous souhaiterions approfondir notre analyse des corrélations entre les événements $\mathrm{du}$ jeu et les constructions syntaxiques particulières. Aux niveaux syntaxique et lexical, nous voudrions analyser notamment la compétition entre des expressions qui ont la même référence mais des structures syntaxiques et des tailles différentes, par exemple équipe de France vs les Français, ou encore David Skrela vs Skrela. Parallèlement, il serait intéressant d'étudier la variété et la fréquence des mots utilisés en rapport avec les contraintes de temps.

Par ailleurs, nous n'avons pas pour l'instant analysé l'influence de la contrainte «émotion », Max(Emo), sur le discours, notamment au niveau prosodique. Toutefois, nous sommes conscients que le commentaire sportif n'a pas qu'une visée informationnelle, qui se résumerait à dire ce qu'il y a à l'image. Au contraire, le commentaire a essentiellement une visée émotionnelle, qui s'appuie et sur l'image et sur le discours. Cette dimension est particulièrement apparente dans le commentaire d'événements qui n'ont pas directement d'importance par rapport au jeu, comme par exemple le temps des hymnes. Le commentaire des hymnes n'apporte aucune information utile pour le jeu, mais permet en revanche de construire cette dimension «émotion ». La contrainte $\operatorname{Max}(\mathrm{Emo})$ affecte la prosodie de l'ensemble du discours, en influant à un niveau macro sur l'étendue de voix du locuteur (cf. §2.3.). A un niveau plus micro, nous distinguons comme Audrit et al. (2012), des périodes de jeu où « le suspense se construit » (building up suspense), qui présentent un patron mélodique spécifique, qui fera l'objet d'une analyse systématique. Nous vérifierons ainsi, dans ces moments de tension, l'influence des contraintes simultanées de la présence d'un événement marquant Fid(EvM), et d'une fenêtre d'anticipation suffisante sur la F0 et les variations mélodiques (Suspense). 
Ces perspectives s'inscrivent directement dans le prolongement de ce travail, dont le propos est d'observer les relations entre le phonogenre commentaire sportif, les différents sports concernés, et les énoncés produits effectivement.

\section{Références bibliographiques}

Apothéloz,D. (1997). Les dislocations à gauche et à droite dans la construction des schématisations, in Berrendonner, A. \& Miéville, D. (eds.), Logique, discours et pensée. Mélanges offerts à Jean-Blaise Grize, Berne: Peter Lang, 183-217.

Ashby, W. J. (1988). The Syntax, Pragmatics, and Sociolinguistics of Left and Right Dislocations in French, Lingua 75, 203-229.

Audrit, S., Pršir, T., Auchlin, A. \& Goldman, J.-P. (2012). Sport in the media: a contrasted study of three sport live media reports with semiautomatic Tools, in Qiuwu, M., Hongwei, D. \& Hirst, D. (eds.), Proceedings of the 6th International Conference on Speech Prosody, vol. 1, Shanghai : Tongji University Press, 127-130, [http://www. speechprosody2012.org/page.asp?id=157].

Augendre, S. \& Mathon, C. (2012). Comment l'information à transmettre influence-t-elle les constructions référentielle et syntaxique du commentaire sportif en direct ?, Actes du CMLF 2012 - 3ème Congrès Mondial de Linguistique Française, Paris : EDP Sciences, 2027-2040, [http://dx.doi.org/10.1051/shsconf/20120100274].

C. Barras, E. Geoffrois, Z. Wu, and M. Liberman (2000), Transcriber: development and use of a tool for assisting Speech corpora production, Speech Communication special issue on Speech Annotation and Corpus Tools, Vol 33, No 1-2, January 2000.

Deulofeu, J. (2000). Les commentaires sportifs constituent-ils un "genre", au sens linguistique du terme ?, in Bilger, M. (ed.), Corpus: Méthodologie et applications linguistiques, Paris : Champion.

Kerbrat-Orecchioni, C. (1990). Les interactions verbales. Paris : Armand-Colin.

Martin, P. (2000). WinPitch 2000 : a tool for experimental phonology and intonation research, in Proceedings of the Prosody 2000 Workshop, Kraków, 2-5 Octobre 2000.

Mathon, C. \& Boulakia, G. (2009). Le commentaire sportif en direct : une combinatoire de différentes fonctions de la prosodie, in Yoo, H.-Y. \& Delais-Roussarie, E. (eds.), Actes d'IDP 2009, Paris, ISSN 2114-7612, 287-301.

Prince, A. \& Smolensky, P. (1993). Optimality Theory: Constraint Interaction in Generative Grammar. Blackwell Publishers.

Pršir, T., Goldman, J.-P. \& Auchlin, A. (2013). Variation prosodique situationnelle : étude sur corpus de huit phonogenres en français, in Mertens, P. \& Simon, A.C. (eds.), Proceedings of the Prosody-Discourse Interface Conference 2013 (IDP-2013). Leuven, September 11-13, 2013, 107-112, [http://wwwling.arts.kuleuven.be/franitalco/idp2013/Proceedings.html].

${ }^{1}$ D'autres hiérarchisations sont plus sensibles pour d'autres sports. Par exemple, le fait que la contrainte qui maximise l'émotion $\operatorname{Max}(\mathrm{Emo})$ domine la contrainte qui maximise le discours complémentaire Max(Comp) est particulièrement sensible pour le patinage artistique, où les commentateurs sont souvent amenés à se taire pour laisser libre cours à l'émotion portée par la musique. 